\title{
The cubic root unscented kalman filter to estimate the position and orientation of mobile robot trajectory
}

Omar Bayasli, Hessen Salhi

Department of Electronic, University of Blida 1, Algeria

\begin{tabular}{l}
\hline \hline Article Info \\
\hline Article history: \\
Received Nov 30, 2019 \\
Revised Apr 26, 2020 \\
Accepted May 6, 2020 \\
\hline
\end{tabular}

Keywords:

CRUKF

Mobile robot

Nonlinear system

Sensor

UKF

\begin{abstract}
In this paper we introduce a cubic root unscented kalman filter (CRUKF) compared to the unscented kalman filter (UKF) for calculating the covariance cubic matrix and covariance matrix within a sensor fusion algorithm to estimate the measurements of an omnidirectional mobile robot trajectory. We study the fusion of the data obtained by the position and orientation with a good precision to localize the robot in an external medium; we apply the techniques of kalman filter (KF) to the estimation of the trajectory. We suppose a movement of mobile robot on a plan in two dimensions. The sensor approach is based on the CRUKF and too on the standard UKF which are modified to handle measurements from the position and orientation. A real-time implementation is done on a three-wheeled omnidirectional mobile robot, using a dynamic model with trajectories. The algorithm is analyzed and validated with simulations.
\end{abstract}

Copyright $\odot 2020$ Institute of Advanced Engineering and Science. All rights reserved.

Corresponding Author:

Omar Bayasli,

Department of Electronic,

University Blida 1,

B.P.270, Road of Soumaa, Blida, Algeria.

Email: bayaomar97@gmail.com

\section{INTRODUCTION}

The mobile robots are equipped with sensors to measure the distance of the robot from the space of the objects where they move. Measurements which are always linked by noises are then fused together in a filter to obtain an estimate of the position and orientation of the mobile robot trajectory in the space and plan $[1,2]$. The most important problems related to robots are situated in sensors powere by their battery which reduces the autonomy. Also there are situations where sensors cannot function simultaneously, when they use the same frequency band [3, 4]. The Kalman filter is an optimal linear estimator when the process noise and the measurement noise can be modeled by white Gaussian noise. Non-linear problems can be solved with the unscented Kalman filter (UKF) and we propose a new algorithm that is called the cubic root unscented Kalman filter (CRUKF) to solve also nonlinear problems. In our work, we use this technique to estimate the position and the angle of the robot in its displacement then a comparison between the performances of the two filters is presented to give an evaluation of more precised measurement and will appreciate better statistical properties $[2,5]$.

This paper is organized as follow; we present the modeling of the mobile robot with the equations of trajectory and angles of the robot. Next, we pass to the presentation of the UKF algorithm and we propose a CRUKF algorithm to improve the accuracy of the state estimate. Finally we present and discuss the simulation results and outline some possible extensions for future investigations.

\section{MODELING OF MOBILE ROBOT}

The robot takes the position trajectory $\mathrm{T}$ between two moments from sampling with the variation in the position and the direction then we supposes in our paper the omnidirectional mobile robot like shown in 
Figure 1. Our robot have three wheels whoos effect on the robot speed because of their weight and friction on the ground, we suppose $m_{1}, m_{2}$ and $m_{3}$ the robot mass applied on the wheels. The omnidirectional mobile Robot has two independently speed-controlled wheels equipped with sensors, and a castor wheel [2, 6-12]. For such a robot, an approximated, discrete-time model is:

$$
\begin{gathered}
A_{k+1}=A_{k}+v_{k} \cdot m_{k} \cdot t \cdot u_{1} \cdot \cos \left(\theta_{k+1}\right)+w_{1} \\
B_{k+1}=B_{k}+v_{k} \cdot m_{k} \cdot t \cdot u_{2} \cdot \sin \left(\theta_{k+1}\right)+w_{2} \\
\theta_{k+1}=\theta_{k}+\Delta \theta+w_{3}
\end{gathered}
$$

where $\left(A_{k}, B_{k}\right)$ is the position of the robot at time $t_{k}$, with $A_{k}=\left(\begin{array}{c}A_{1} \\ A_{2} \\ A_{3}\end{array}\right), A_{k}=A_{1}, B_{k}=A_{2}, \theta_{k}=A_{3}$, $\theta_{k}$ represente the angle between the robot axle and the x-axis, $w_{k}=\left(\begin{array}{c}w_{1} \\ w_{2} \\ w_{3}\end{array}\right)$ are zero-mean uncorrelated Gaussian noises and $u_{k}=\left(\begin{array}{l}u_{1} \\ u_{2}\end{array}\right)$ are the angular velocities of wheels, $m_{k}=R \cdot\left(m_{1}+m_{2}+m_{3}\right) / 3 L$ is the mass of the robot in wheels. $v_{k}=R \cdot\left(u_{1}+u_{2}\right) / 2$ is the linear velocity of the robot, $t=t_{k+1}-t_{k}$ is the sampling period and $\Delta \theta=R .\left(u_{2}-u_{1}\right) \cdot t / 2 L$ is the rotation within $\left[t_{k}, t_{k+1}\right] . R$ is the radius of the wheels and $L$ is the length of the axes.

The omnidirectional mobile robot is equipped with sensors to measure its position and its orientation like shown in Figure 2. The figure also defines the environment of the robot, assumed perfectly known $[7,10,13]$. In our work we propose the trajectory $\mathrm{T}$ on which we apply the technical of Kalman filters CRUKF and UKF to estimate this trajectory.

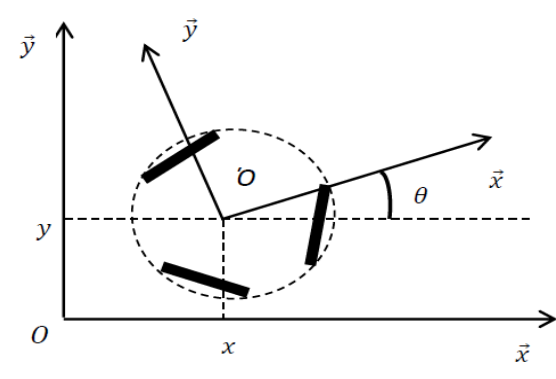

Figure 1. Omnidirectional mobile robot

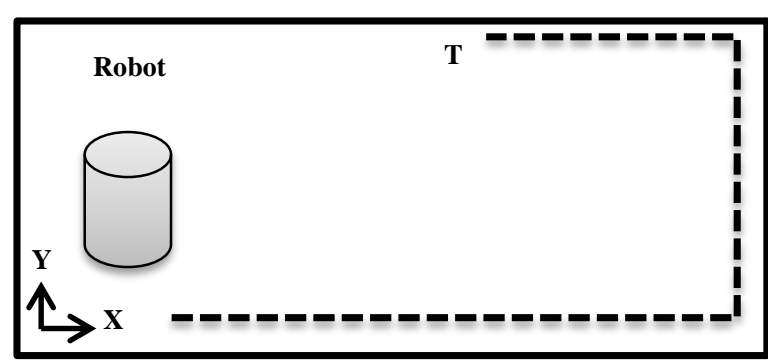

Figure 2. Omnidirectional mobile robot trajectory $\mathrm{T}$

\subsection{The equations of trajectory $T$}

The trajectory of the robot is defined by the equation $B_{k+1}=f\left(A_{k+1}\right)$ where $A_{k}=A_{1}=1$; $B_{k}=A_{2}=1 ; \theta_{k}=A_{3}=1 ; t=1 s ; v_{1}=v_{2}=0,02 \mathrm{~m} / \mathrm{s} ; u_{1}=0,2 ; u_{2}=0,1 ; \Delta \theta=\pi / 2 ; m_{1}=0,3 \mathrm{~g}$; $m_{2}=0,3 \mathrm{~g} ; \quad m_{3}=0,3 \mathrm{~g} ; R=0,5 \cdot 10^{-2} \mathrm{~m} \quad L=0,18 \mathrm{~m} \quad$ and $\quad m_{k}=R .\left(m_{1}+m_{2}+m_{3}\right) / 3 \mathrm{~L}$; then $m_{k}=0,0083 \mathrm{~g}$.

We have our model:

$$
\left\{\begin{array}{c}
A_{k+1}=A_{k}+v_{1} m_{k} t u_{1} \cos \left(\theta_{k+1}\right)+w_{1} \\
B_{k+1}=B_{k}+v_{2} m_{k} t u_{2} \sin \left(\theta_{k+1}\right)+w_{2} \\
\theta_{k+1}=\theta_{k}+\Delta \theta+w_{3}
\end{array}\right.
$$

then

$$
\left\{\begin{array}{l}
A_{k+1}-A_{k}-w_{1}=v_{1} m_{k} t u_{1} \cos \left(\theta_{k+1}\right) \\
B_{k+1}-B_{k}-w_{2}=v_{2} m_{k} t u_{2} \sin \left(\theta_{k+1}\right)
\end{array}\right.
$$

then

$$
\frac{\left(A_{k+1}-A_{k}-w_{1}\right)}{v_{1} m_{k} t u_{1}}=\cos \left(\theta_{k+1}\right)
$$




$$
\frac{\left(B_{k+1}-B_{k}-w_{2}\right)}{v_{2} m_{k} t u_{2}}=\sin \left(\theta_{k+1}\right)
$$

and we make square:

$$
\begin{aligned}
& \frac{\left(A_{k+1}-A_{k}-w_{1}\right)^{2}}{\left(v_{1} m_{k} t u_{1}\right)^{2}}=\cos ^{2}\left(\theta_{k+1}\right) \\
& \frac{\left(B_{k+1}-B_{k}-w_{2}\right)^{2}}{\left(v_{2} m_{k} t u_{2}\right)^{2}}=\sin ^{2}\left(\theta_{k+1}\right) \\
& (4)+(5) \Rightarrow \frac{\left(A_{\mathrm{k}+1}-\mathrm{A}_{\mathrm{k}}-\mathrm{w}_{1}\right)^{2}}{\left(\mathrm{v}_{1} \mathrm{~m}_{\mathrm{k}} \mathrm{t} \mathrm{u}_{1}\right)^{2}}+\frac{\left(\mathrm{B}_{\mathrm{k}+1}-\mathrm{B}_{\mathrm{k}}-\mathrm{w}_{2}\right)^{2}}{\left(\mathrm{v}_{2} \mathrm{~m}_{\mathrm{k}} \mathrm{tu}\right)^{2}}=\cos ^{2}\left(\theta_{\mathrm{k}+1}\right)+\sin ^{2}\left(\theta_{\mathrm{k}+1}\right)
\end{aligned}
$$

then

$$
\begin{aligned}
& \frac{\left(A_{k+1}-A_{k}-w_{1}\right)^{2}}{\left(v_{1} m_{k} t u_{1}\right)^{2}}+\frac{\left(B_{k+1}-B_{k}-w_{2}\right)^{2}}{\left(v_{2} m_{k} t u_{2}\right)^{2}}=1 \\
& \Rightarrow \frac{\left(B_{k+1}-B_{k}-w_{2}\right)^{2}}{\left(v_{2} m_{k} t u_{2}\right)^{2}}=1-\frac{\left(A_{k+1}-A_{k}-w_{1}\right)^{2}}{\left(v_{1} m_{k} t u_{1}\right)^{2}} \\
& \Rightarrow\left(B_{k+1}-B_{k}-w_{2}\right)^{2}=\left(v_{2} m_{k} t u_{2}\right)^{2}\left(1-\frac{\left(B_{k+1}-B_{k}-w_{1}\right)^{2}}{\left(v_{1} m_{k} t u_{1}\right)^{2}}\right) \\
& \Rightarrow B_{k+1}-B_{k}-w_{2}=\sqrt{\left(v_{2} m_{k} t u_{2}\right)^{2}\left(1-\frac{\left(A_{k+1}-A_{k}-w_{1}\right)^{2}}{\left(v_{1} m_{k} t u_{1}\right)^{2}}\right)}
\end{aligned}
$$

then

$$
B_{k+1}=B_{k}+w_{2}+v_{2} m_{k} t u_{2} \sqrt{\left(1-\frac{\left(A_{k+1}-A_{k}-w_{1}\right)^{2}}{\left(v_{1} m_{k} t u_{1}\right)^{2}}\right)}
$$

For the trajectory T we take: $w_{1}=0, w_{2}=0$ and $w_{3}=0$

$$
\mathrm{B}_{\mathrm{k}+1}=1+0+0,02 \cdot 1 \cdot 0,0083 \cdot 0,1 \cdot \sqrt{\left(1-\frac{\left(\mathrm{A}_{\mathrm{k}+1}-1-0\right)^{2}}{(0,02 \cdot 10,0083 . .0,2)^{2}}\right)}
$$

Then the final equation of $\mathrm{T}$ is:

$$
\mathrm{B}_{\mathrm{k}+1}=1+0,016 \cdot 10^{-3} \sqrt{\left(1-\frac{\left(\mathrm{A}_{\mathrm{k}+1}-1\right)^{2}}{0,256 \cdot 10^{-6}}\right)}
$$

\subsection{The angle of robot $\theta_{\mathrm{k}+1}$}

We have

$$
\left\{\begin{array}{l}
\frac{\left(A_{k+1}-A_{k}-w_{1}\right)}{v_{1} m_{k} t u_{1}}=\cos \left(\theta_{k+1}\right) \\
\frac{\left(B_{k+1}-B_{k}-w_{2}\right)}{v_{2} m_{k} t u_{2}}=\sin \left(\theta_{k+1}\right)
\end{array}\right.
$$

(2) $\Rightarrow \theta_{k+1}=\operatorname{acos}\left(\frac{A_{k+1}-A_{k}-w_{1}}{v_{1} m_{k} t u_{1}}\right)$ is the angle of robot with axis, or can we find this angle by (3): 
(3) $\Rightarrow \frac{\left(B_{k+1}-B_{k}-w_{2}\right)}{v_{2} m_{k} t u_{2}}=\sin \left(\theta_{k+1}\right) \Rightarrow \theta_{k+1}=\operatorname{asin}\left(\frac{B_{k+1}-B_{k}-w_{2}}{v_{2} m_{k} t u_{2}}\right)$

$\theta_{k}=\operatorname{atan}\left(\frac{\operatorname{imag}\left(\theta_{k+1}\right)}{\operatorname{real}\left(\theta_{k+1}\right)}\right)$ is the angle of robot with wheels axis

\section{UNSCENTED KALMAN FILTER AND HER ALGORITHM}

The UKF has been developed in recent years to overcome two main problems of the EKF (extended Kalman filter), the poor approximation properties of the first order approximation and the requirement for the noises to be Gaussian. The basic idea behind the UKF is that of finding a transformation that allows approximating the mean and the covariance of a random vector of length $n$ when it is transformed by a nonlinear map. This is done by computing a set of $2 n+1$ points, called sigma points, on the basis of the mean and variance of the original vector, transforming these points by the nonlinear map and then approximating the mean and variance of the transformed vector from the transformed sigma points. We refer to $[2-5,14-17]$ for the theoretical aspects.

As for approximating properties of the filter, it has been shown that, while the EKF estimate of the state is accurate to the first order, the UKF estimate is accurate to the third order in the case of Gaussian noises. The covariance estimate also is accurate to the first order for the EKF, and to the second order for the UKF and her algorithm is:

$$
\begin{aligned}
& \text { UKF Algorithm } \\
& \text { - Initialization: } \\
& \hat{A}_{0}=E\left[A_{0}\right], P_{A_{0}}=E\left[\left(A_{0}-\hat{A}_{0}\right)\left(A_{0}-\hat{A}_{0}\right)^{T}\right] \\
& \hat{A}_{0}^{a}=E\left[\hat{A}_{0}^{a}\right]=\left[\begin{array}{lll}
\hat{A}_{0}^{T} & \bar{v}_{0}^{T} & \bar{n}_{0}^{T}
\end{array}\right]^{T} \\
& P_{0}^{a}=E\left[\left(A_{0}^{a}-\hat{A}_{0}^{a}\right)\left(A_{0}^{a}-\hat{A}_{0}^{a}\right)\right]^{T}=\left[\begin{array}{ccc}
P_{A_{0}} & 0 & 0 \\
0 & R_{v} & 0 \\
0 & 0 & R_{n}
\end{array}\right] \\
& \text { For } \mathrm{k}_{u}=1, \ldots, \infty \text { : } \\
& \text { 1. Set } t_{u}=k_{u}-1 \quad \text { (for unscented Kalman filter) } \\
& \text { 2. Calculate sigma-points: }
\end{aligned}
$$

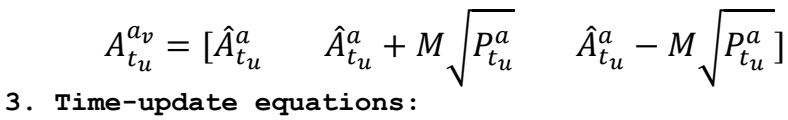

$$
\begin{aligned}
& A_{k_{u} \mid t_{u}}^{x}=f\left(A_{t_{u}}^{x}, A_{t_{u}}^{v}, u_{t_{u}}\right) \\
& \hat{x}_{k_{u}}^{s}=\sum_{i=0}^{2 L} w_{i}^{m} A_{i, k_{u} \mid t_{u}}^{x} \\
& P_{x_{k_{u}}}^{s}=\sum_{i=0}^{2 L} w_{i}^{c}\left(A_{i, k_{u} \mid t_{u}}^{x}-\hat{x}_{k_{u}}^{s}\right)\left(A_{i, k_{u} \mid t_{u}}^{x}-\hat{x}_{k_{u}}^{s}\right)^{T}
\end{aligned}
$$

\section{THE CUBIC ROOT UKF (CRUKF) PROPOSITION}

In our paper we propose the cubic root CRUKF algorithm which is based on square root UKF algorithm $[18,19]$. So we use in our algorithm a proposition of new function $q q(x)$ and UKF algorithm [4]. We study the coefficients of covariance matrix to introduce the cubic root. In this technique, we based on the same principals of the UKF algorithm.

\subsection{The function $q q(x)$ proposition}

The new function $q q(x)$ is the quadrature quadrature or orthogonal orthogonal function for find and calculates two matrixes quadrature or orthogonal and its equation is: 


$$
q q=I_{1}-\frac{2 V_{1} V_{1}^{T}}{\left\|V_{1}\right\|} \cdot I_{2}-\frac{2 V_{2} V_{2}^{T}}{\left\|V_{2}\right\|}
$$

$I_{1}$ and $I_{2}$ are identity matrix

$V_{1}$ and $V_{2}$ are vectors ; $V_{1}^{T}$ and $V_{2}^{T}$ are vectors transposes

$\left\|V_{1}\right\|$ and $\left\|V_{2}\right\|$ vectors modules

\subsection{The CRUKF algorithm}

CRUKF Algorithm

- Initialization:

$$
\hat{A}_{0}=E\left[A_{0}\right], \quad S_{A_{0}}=\sqrt[3]{E\left[\left(A_{0}-\hat{A}_{0}\right)\left(A_{0}-\hat{A}_{0}\right)^{T}\right]}, \quad S_{v}=\sqrt[3]{R_{v}} \text { and } S_{n}=\sqrt[3]{R_{n}}
$$

$\hat{A}_{0}^{a}=E\left[A^{a}\right]=\left[\begin{array}{lll}\hat{A}_{0} & \bar{v} & \bar{n}\end{array}\right]^{T}$

For $k_{c}=1, \ldots, \infty$ :

$$
S_{0}^{a}=\sqrt[3]{E\left[\left(A_{0}^{a}-\hat{A}_{0}^{a}\right)\left(A_{0}^{a}-\hat{A}_{0}^{a}\right)^{T}\right]}, \quad \text { then } S_{0}^{a}=\left[\begin{array}{ccc}
S_{A_{0}} & 0 & 0 \\
0 & S_{v} & 0 \\
0 & 0 & S_{n}
\end{array}\right]
$$

1. Set $t_{c}=k_{c}-1 ;$ (for the cubic root unscented Kalman filter)

2. Calculate sigma-points for time-update:

$A_{t_{c}}^{a}=\left[\begin{array}{lll}\hat{A}_{t_{c}}^{a} & \hat{A}_{t_{c}}^{a}+N S_{A_{t_{c}}}^{a} & \hat{A}_{t_{c}}^{a}-N S_{A_{t_{c}}}^{a}\end{array}\right]$

3. Time-update equations:

$A_{k_{c} \mid t_{c}}^{x}=f\left(A_{t_{c}}^{a}, A_{t_{c}}^{v}, u_{t_{c}}\right)$

$\hat{x}_{k_{c}}^{s}=\sum_{i=0}^{2 L} w_{i}^{m} A_{i, k_{c} \mid t_{c}}^{x}$

$S_{x_{k_{c}}}^{s}=q q\left\{\left[\sqrt[3]{w_{1}^{c}}\left(A_{1: 2 L, k_{c} \mid t_{c}}^{x}-\hat{x}_{k_{c}}^{S}\right)\right]\right\} \quad ; \quad$ (quadrature quadrature function)

$S_{x_{k_{c}}}^{s}=$ update $\left\{S_{x_{k_{c}}}^{s}, A_{0, k_{c} \mid t_{c}}^{x}-\hat{x}_{k_{c}}^{s}, w_{0}^{(c)}\right\}$; time update

$B_{k_{c} \mid t_{c}}=h\left(A_{i, k_{c} \mid t_{c}}^{x} A_{t_{c}}^{n}\right)$

$\hat{B}_{k_{c}}^{s}=\sum_{i=0}^{2 L} w_{i}^{m} B_{i, k_{c} \mid t_{c}}$

4. Measurement-update equations:

$S_{B_{k_{c}}}^{S}=q q\left\{\left[\sqrt[3]{w_{1}^{c}}\left(B_{1: 2 L, k_{c} \mid t_{c}}-\hat{B}_{k_{c}}^{s}\right)\right]\right\}$

$S_{B_{k_{c}}}^{S}=$ update $\left\{S_{B_{k_{c}}}^{S}, B_{0, k_{c} \mid t_{c}}-\hat{B}_{k_{c}}^{S}, w_{0}^{(c)}\right\}$

$P_{x_{k_{c}} B_{k_{c}}}=\sum_{i=0}^{2 L} w_{i}^{c}\left(A_{i, k_{c} \mid t_{c}}^{x}-\hat{x}_{k_{c}}^{s}\right)\left(B_{i, k_{c} \mid t_{c}}-\hat{B}_{k_{c}}^{s}\right)^{T}$

$G_{k_{c}}=\left(P_{x_{k_{c}} B_{k_{c}}} / S_{B_{k_{c}}}^{T}\right) / S_{B_{k_{c}}}^{S}$

$\hat{A}_{k_{c}}=\hat{A}_{k_{c}}^{s}+G_{k_{c}}\left(B_{k_{c}}-\hat{B}_{k_{c}}^{s}\right)$

$U=G_{k} S_{B_{k_{c}}}^{S}$

$S_{x_{k_{c}}}=$ update $\left\{S_{x_{k_{c}}}^{s}, U,-1\right\}$

We have two filters UKF and CRUKF where: $M=\sqrt{L+\lambda}, \quad N=\sqrt[3]{L+\lambda}, w_{0}^{m}=\lambda /(L+\lambda)$, $w_{0}^{c}=w_{0}^{m}+\left(1+\alpha^{2}+\beta\right), w_{i}^{c}=w_{i}^{m}=1 / 2(L+\lambda)$, for $i=1 \ldots 2 L$, and $\lambda=\alpha^{2}(L+k)$ is a compound scaling parameter. $L$ is the dimension of the augmented state-vector $0<\alpha<1$ is the primary scaling factor determining the extent of the spread of the sigma-points around the prior mean. Typical range for $\alpha$ is $1 e-3<\alpha<1 . \beta$ is a secondary scaling factor used to emphasize the weighting on the zeros sigma-point for the posterior covariance calculation. $\beta$ can be used to minimize certain higher-order error terms based on known moments of the prior $R_{v}$. For Gaussian priors, $\beta=2$ is optimal. $k$ is a tertiary scaling factor and is usually set equal to 0 . In general, the optimal values of these scaling parameters will be a specific problem $[2,18,19,20-25]$.

General notes:

The augmented state vector and sigma-point vector is given by: $\mathrm{x}^{\mathrm{a}}=\left[\begin{array}{lll}\mathrm{x}^{\mathrm{T}} & \mathrm{v}^{\mathrm{T}} \mathrm{n}^{\mathrm{T}}\end{array}\right]^{\mathrm{T}}$, $A^{a}=\left[\begin{array}{lll}\left(A^{x}\right)^{T} & \left(A^{v}\right)^{T} & \left(A^{n}\right)^{T}\end{array}\right]^{T}$ where $R_{v}$ and $R_{n}$ are the process-noise and observation-noise covariance matrices.

Linear-algebra operators:

$\sqrt[3]{.}$ : matrix cubic-root using lower triangular.

$q q(X)$ : lower-orthogonal part of $X$ matrix resulting from function $\operatorname{ortho}(X)$ decomposition of data-matrix $X$. update $\left\{R, U, \pm^{+} v\right\}: N$ consecutive rank-1 updates of the lower-orthogonal factor $q$ by the $N$ columns of

The cubic root unscented Kalman filter to estimate the position and orientation... (Omar Bayasli) 
$\sqrt{v} U:$. / : Efficient least-squares pseudo inverse implemented using orthogonal $Q Q$ decomposition with pivoting finally this parameters for two filters CRUKF and UKF.

\section{SIMULATION RESULTS}

We compare the performance of CRUKF and UKF in our paper, we starte from the point of trajectory $\mathrm{T}:\left(A_{0}, B_{0}\right)=(1,1)$ with $\theta_{0}=0$. In the chosen sample time $t=1 \mathrm{~s}$ and each trajectory has been completed in $k_{f}=400 \mathrm{~s}$, imposing a profile to the angular velocities of the wheels. The parameters and weights used in the CRUKF and UKF are: $=10^{-4} ; \beta=5 ; \kappa=(8-n) / 2 ; \lambda=\alpha^{2}(n+\kappa)-n$. $\beta=3$ minimizes the error of the a posteriori covariance when the random vector is Gaussian.

- $W=\operatorname{diag}\left\{\begin{array}{lll}0.123 & 0.124 & 0.121\end{array}\right\}$, which corresponds to a standard deviation less than one centimeter on $A_{k}$ and $B_{k}$ and less than one degree on $\theta_{k}$;

- $\quad V=\operatorname{diag}\left\{\begin{array}{lll}0.110 & 0.100 & 0.111\end{array}\right\}$, a standard deviation less that 0.1 centimeter for each sensor;

- $\hat{A}_{0 / 0}=\left[\begin{array}{lll}1.5000 & 1.9821 & 2.4123\end{array}\right]^{\prime}$, the initial estimate state of robot

- $A_{0}=\left[\begin{array}{lll}1 & 1 & 0\end{array}\right]^{\prime}$, the initial state of robot

- $P_{0 / 0}=\left[\begin{array}{lll}0.5010 & 0.5021 & 0.5023\end{array}\right]^{\prime}$, a standard deviation of width and length on the position and standard orientation.

We compare the performance of the two filters we have computed

- The average error position index: $\varepsilon_{x}=\frac{1}{k_{f}+1} \sum_{k=0}^{k_{f}}\left\|\left[A_{k}-\hat{A}_{k / k}, B_{k}-\hat{B}_{k / k}\right]^{\prime}\right\|$

- The average orientation position index: $\varepsilon_{\theta}=\frac{1}{k_{f}+1} \sum_{k=0}^{K_{f}}\left|\theta_{k+1}-\hat{\theta}_{k+1 / k}\right|$

- The estimation error covariance averaged along the whole trajectory has been computed: $\pi=\frac{1}{k_{f}+1} \sum_{k=0}^{k_{f}} \operatorname{tr}\left(\sum P_{k / k}\right)$

The comparison between CRUKF and UKF at our case as follows: we start with time, we take three values for $k$ to each algorithm. Table 1 show the comparison and the simulation results show in different figures: Figures 3-6.

\subsection{Estimation of position by CRUKF and UKF}

Figures 3 and 4 show the simulation results using the UKF and CRUKF applied to the trajectory estimation and errors. Figure 3 shows the robot trajectory with its estimate using UKF (red) compared to the CRUKF (bleu), the poursuit was well done. Figure 4 presents the estimation error between the original trajectory and both of the two filters also the MSE (mean square error) of the algorithems, it can be seen the CRUKF was better then the UKF.

Table 1. Comparison between CRUKF and UKF

\begin{tabular}{|c|c|c|c|c|c|c|c|c|c|}
\hline Algorithm & & $\varepsilon_{\mathrm{x}}(\mathrm{m})$ & & & $\varepsilon_{\theta}(\mathrm{rad})$ & & & $\pi$ & \\
\hline CRUKF & 0.0156 & 0.0258 & 0.0077 & 0.3550 & 0.4405 & 0.1815 & 0.0263 & 0.0116 & 0.0104 \\
\hline UKF & 0.0082 & 0.0050 & 0.0074 & 0.1563 & 0.0716 & 0.0493 & 0.0015 & 0.0010 & 0.0003 \\
\hline
\end{tabular}

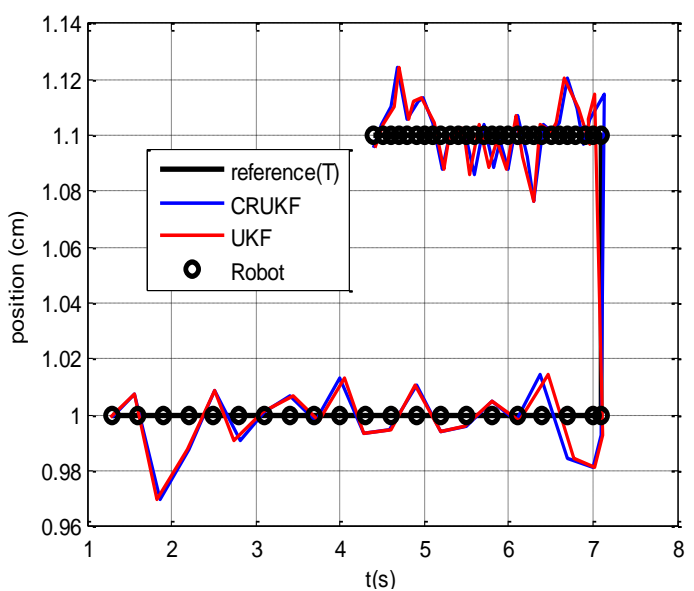

Figure 3. Estimate reference trajectory $\mathrm{T}$ by CRUKF and UKF
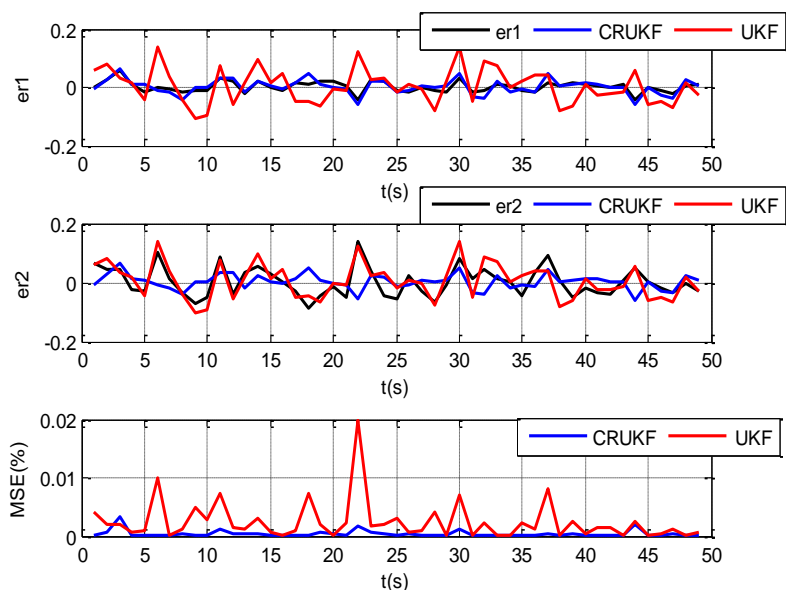

Figure 4. Estimate error er1, er2 and her estimation by CRUKF and UKF and MSE 


\subsection{Estimation of angle by CRUKF and UKF}

Figure 5 shows the angle estimate of the robot using the two filters. Figure 6 exhibits estimation errors of these filters where the UKF was here better than the CRUKF.

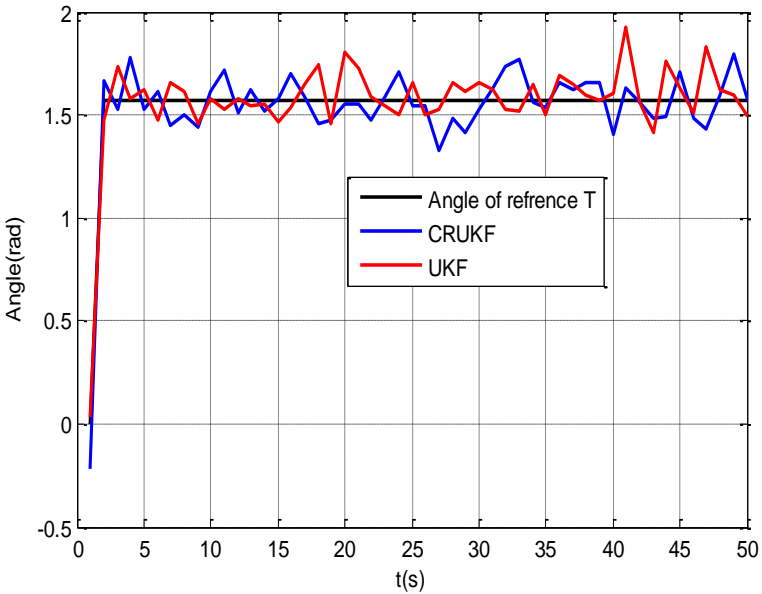

Figure 5. The estimate angle of trajectory $\mathrm{T}$ with axis by CRUKF and UKF
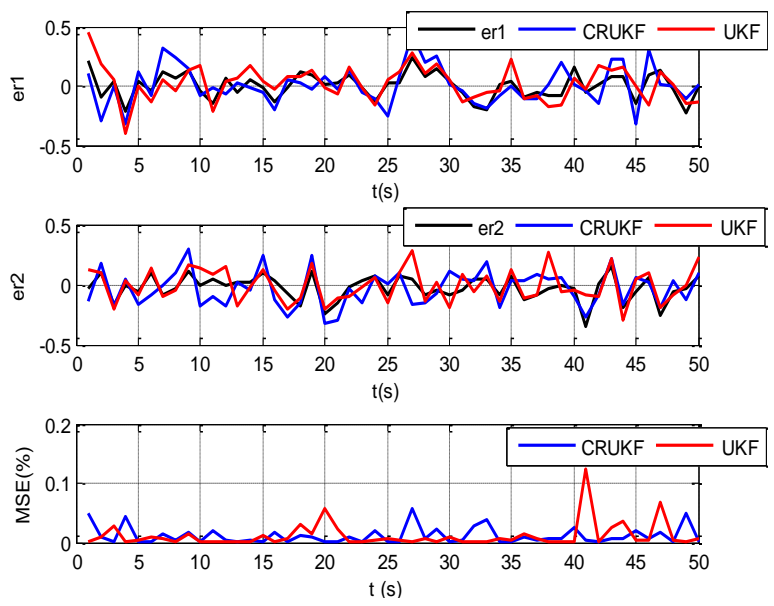

Figure 6. Estimate error er1,er2 of angle and her estimation by CRUKF and UKF and MSE

\section{CONCLUSION}

The analysis of the results in Table 1 is that the two filters perform comparably the position and orientation of the robot, we see that the estimation error of UKF is weak than those the estimation error of CRUKF in angle estimation contrary in the position where the CRUKF was better because the approximating properties of each filter. These two filters, always giving a value of the error covariance $\pi$ of index which is better than those given by any sensor, and completely close to that obtained when all the sensors are used. Results shows the estimate reference trajectory $\mathrm{T}$ and estimate error by CRUKF and UKF too MSE of this case and the estimate of the robot angle and its estimate error by CRUKF and UKF too MSE of this case, we see that the estimations were optimal. In the near future this technique of work can be done and develop on other types of robots and other applications with less sensor but with a great measuring accuracy in realtime but its better to make one filter complete the other.

\section{REFERENCES}

[1] L. D'Alfonso, et al., "Mobile robot localization via EKF and UKF: A comparison based on real data," Robotics and Autonomous Systems, vol. 74, part A, pp. 122-27, 2015.

[2] L. D'Alfonso, et al., "Filters for mobile robots: EKF, UKF and sensor switching - experimental results," 20119 th IEEE International Conference on Control and Automation (ICCA), Santiago, pp. 925-930, 2011.

[3] E. A. Wan and R. Van Der Merwe, "The unscented Kalman filter for nonlinear estimation," Proceedings of the IEEE 2000 Adaptive Systems for Signal Processing, Communications, and Control Symposium (Cat. No.00EX373), Lake Louise, Alberta, Canada, pp. 153-158, 2000.

[4] S. J. Julier and J. K. Uhlmann, "Unscented filtering and nonlinear estimation," in Proceedings of the IEEE, vol. 92, no. 3, pp. 401-422, Mar. 2004

[5] M. St-Pierre and D. Gingras, "Comparison between the unscented Kalman filter and the extended Kalman filter for the position estimation module of an integrated navigation information system," IEEE Intelligent Vehicles Symposium, 2004, Parma, Italy, pp. 831-835, 2004.

[6] H. Harianto, M. Rivai, D. Purwanto, "Implementation of Electronic Nose in Omni-directional Robot," International Journal of Electrical and Computer Engineering (IJECE), vol. 3, no. 3, pp. 399-406, 2013.

[7] M. Hamissi and K. Faez, "Real-Time Hand Gesture Recognition Based on the Depth Map for Human Robot Interaction," International Journal of Electrical and Computer Engineering (IJECE), vol. 3, no. 6, pp. 770-778, 2013.

[8] O. Bachir and A.-F. Zoubir, "Adaptive Neuro-fuzzy Inference System Based Control of puma 600 Robot Manipulator," International Journal of Electrical and Computer Engineering (IJECE), vol. 2, no. 1, pp. 90-97, 2011.

[9] B. J. Stephens and C. G. Atkeson, "Push Recovery by stepping for humanoid robots with force controlled joints," 2010 10th IEEE-RAS International Conference on Humanoid Robots, Nashville, TN, pp. 52-59, 2010. 
[10] A. Rezaee and R. Afshar, "Digital Encoder Designing for Mobile Robot Control," International Journal of Electrical and Computer Engineering (IJECE), vol. 4, no. 5, pp. 648-657, 2014.

[11] J. Sahu, B. B. Choudhury, and M. R. Patra, "An Effective Development and Analysis of a Mobile Robot," International Journal of Electrical and Computer Engineering (IJECE), vol. 6, no. 2, pp. 654-665, 2016.

[12] S. Nurmaini and B. Tutuko, "Intelligent Robotics Navigation System: Problems, Methods, and Algorithm," International Journal of Electrical and Computer Engineering (IJECE), vol. 7, no. 6, pp. 3711-3726, 2017.

[13] S. M. Malagon-Soldara, M. Toledano-Ayala, G. Soto-Zarazua, R. V. Carrillo-Serrano, E. A. Rivas-Araiza, "Mobile Robot Localization: A Review of Probabilistic Map-Based Techniques," IAES International Journal of Robotics and Automation (IJRA), vol. 4, no. 1, pp. 73-81, 2015.

[14] S. J. Julier and J. K. Uhlmann, "Reduced sigma point filters for the propagation of means and covariances through nonlinear transformations," Proceedings of the 2002 American Control Conference (IEEE Cat. No.CH37301), Anchorage, AK, USA, vol. 2, pp. 887-892, 2002.

[15] A. Chahmi, M. Bendjebbar, B. Raison, and M. Benbouzid, "An Extender Kalman Filter-based Induction Machines Faults Detection," International Journal of Electrical and Computer Engineering (IJECE), vol. 6, no. 2, pp. 535-548, 2016.

[16] L. F. De Melo and J. F. M. Junior, "Trajectory Planning for Nonholonomic Mobile Robot Using Extended Kalman Filter," Mathematical Problems in Engineering, vol. 2010, pp. 1-22, 2010.

[17] J. Chandrasekar, A. J. Ridley and D. S. Bernstein, "A Comparison of the Extended and Unscented Kalman Filters for Discrete-Time Systems with Nondifferentiable Dynamics," 2007 American Control Conference, New York, NY, pp. 4431-4436, 2007.

[18] R. Van der Merwe and E. A. Wan, "The square-root unscented Kalman filter for state and parameterestimation," 2001 IEEE International Conference on Acoustics, Speech, and Signal Processing. Proceedings (Cat. No.01CH37221), Salt Lake City, UT, USA, vol. 6, pp. 3461-3464, 2001.

[19] Z. Liu, et al., "An improved square root unscented Kalman filter for projectile's attitude determination," 20105 th IEEE Conference on Industrial Electronics and Applications, Taichung, pp. 1747-1751, 2010.

[20] E. A. Wan, R. van der Merwe, and A. T. Nelson, "Dual Estimation and the Unscented Transformation," Neural Information Processing Systems, pp. 666-672, 2000.

[21] J. Andrade-Cetto, T. Vidal-Calleja and A. Sanfeliu, "Unscented Transformation of Vehicle States in SLAM," Proceedings of the 2005 IEEE International Conference on Robotics and Automation, Barcelona, Spain, pp. 323-328, 2005.

[22] S. Sadhu, et al., "Bearing only tracking using square root sigma point Kalman filter," Proceedings of the IEEE INDICON 2004. First India Annual Conference, Kharagpur, India, pp. 66-69, 2004.

[23] R. Kandepu, B. Foss, and L. Imsland, "Applying the unscented Kalman filter for nonlinear state estimation," Journal of Process Control, vol. 18, issue 7-8, pp. 753-768, 2008.

[24] M. Rhudy, et al., "Evaluation of Matrix Square Root Operations for UKF within a UAV GPS/INS Sensor Fusion Application," International Journal of Navigation and Observation, vol. 2011, pp. 1-11, 2011.

[25] M. Rhudy, et al., "Sensitivity and Robustness Analysis of EKF and UKF Design Parameters for GPS/INS Sensor Fusion," AIAA Journal of Aerospace Information Systems, vol. 10, no. 3, pp. 131-143, 2013. 